\title{
FRACTIONAL-VALUED MODAL LOGIC
}

\author{
MARIO PIAZZA \\ Scuola Normale Superiore di Pisa \\ GABRIELE PULCINI \\ Università di Roma "Tor Vergata" \\ and \\ MATTEO TESI \\ Scuola Normale Superiore di Pisa
}

\begin{abstract}
This paper is dedicated to extending and adapting to modal logic the approach of fractional semantics to classical logic. This is a multi-valued semantics governed by pure prooftheoretic considerations, whose truth-values are the rational numbers in the closed interval $[0,1]$. Focusing on the modal logic $K$, the proposed methodology relies on three key components: bilateral sequent calculus, invertibility of the logical rules, and stability (proof-invariance). We show that our semantic analysis of $K$ affords an informational refinement with respect to the standard Kripkean semantics (a new proof of Dugundji's theorem is a case in point) and it raises the prospect of a proof-theoretic semantics for modal logic.
\end{abstract}

§1. Introduction. In a previous paper [24], two of the current authors introduced an informational refinement of the standard Boolean semantics for classical propositional logic. This account reverses the traditional perspective whereby proof-systems are shown to be sound and complete under a preassigned external semantic structure: primacy is instead accorded to proofs which, through their very combinatorial structure, determine from within the semantic value of logical formulas. The intrinsically finitary notion of derivation makes the nature of such semantics finitary as well, despite the infinitude of its truth-values which are elements of the set of rational numbers $\mathbb{Q}$ in the closed interval $[0,1]$. The most visible upshot of the new treatment is the breaking of the symmetry between classical tautologies and contradictions: the former are uniformly interpreted by the value 1 , while the latter become susceptible to different fractional interpretations. This is why we called such semantics "fractional" and we argued that it conforms with the general project of proof-theoretic semantics in considering the classical rules as a medium through which meaning propagates.

Fractional semantics, however, requires an appropriate proof-theoretic platform within which it can be articulated. Namely, in order to allow for a fractional

Received: January 17, 2021.

2020 Mathematics Subject Classification: 03B45, 03F03, 03B50

Keywords and phrases: modal logic, proof theory, general (including proof-theoretic semantics), many-valued logics

(C) The Author(s), 2021. Published by Cambridge University Press on behalf of Association for Symbolic Logic. This is an Open Access article, distributed under the terms of the Creative Commons Attribution licence (https://creativecommons.org/licenses/by/4.0/), which permits unrestricted re-use, distribution, and reproduction in any medium, provided the original work is properly cited. 
interpretation of its formulas, a decidable logic $\mathcal{L}$ needs to be displayed in a sequent system $\mathrm{S}$ (or its variants) meeting three conditions:

(1) Bilateralism: $\mathrm{S}$ proves all the valid formulas of $\mathcal{L}$ and refutes all the invalid ones through logical rules involving both deduction and refutation $[4,10,28$, $29,35]$. In other words, S, as a bilateral system, generates S-derivations for any well-formed formula $A$ of $\mathcal{L}$ : if $A$ is valid, its $\mathrm{S}$-derivation will be an actual proof of $A$; if $A$ is invalid, its S-derivation will provide a formal refutation of $A$, i.e., a proof of its unprovability. ${ }^{1}$

(2) Invertibility: each logical rule of $S$ is invertible, meaning that the provability of its conclusion implies the provability of (each one of) its premise(s) [16]. This means that proof-search in S boils down to an algorithm for decomposing any given sequent into an equivalent multiset of clauses which includes exactly the top-sequents (valid or invalid) of the achieved proof. By such a proof-asdecomposing setting, every S-derivation $\pi$ ending with $A$ shapes a numerical evaluation $\llbracket A \rrbracket$ of $A$. To be more precise, $\llbracket A \rrbracket$ is expressed in terms of the ratio between the number of $\pi$ 's valid top-sequents and the total number of top-sequents.

(3) Stability: two analytic S-proofs with the same end-sequent share the same multiset of top-sequents. This property ensures that fractional evaluations accommodate the demands of an effective semantics: because (bottom-up) proof-search generates a certain multiset of top-sequents, stability can refer directly to such multiset associated with any formula of $\mathcal{L}$.

In sum: on the fractional view, proving a certain sentence $A$ amounts to measuring the quantity of validity involved in $A$, relative to $A$ 's decomposition into elementary top-sequents. In [24] the adopted system for classical propositional logic was the bilateral version of Kleene's sequent calculus $G 4[17,30]$, called $\overline{\overline{\mathrm{G}} 4}$, and satisfying both the invertibility and stability properties. By way of example, in $\overline{\overline{\mathrm{G}} 4}$ the classical contradiction $(p \vee \neg p) \wedge(\neg p \wedge p)$ is thus derivable:

$$
\frac{\frac{\frac{p \Rightarrow p}{p \Rightarrow x}}{\Rightarrow p, \neg p} \Rightarrow \neg \quad \frac{p \Rightarrow}{\Rightarrow \neg p} \Rightarrow \neg \quad \Rightarrow p}{\Rightarrow \neg p \wedge p} \Rightarrow \wedge
$$

The $\overline{\overline{\mathrm{G}} 4}$-derivation above qualifies as a disproof of $(p \vee \neg p) \wedge(\neg p \wedge p)$ and, insofar as it contains only one tautological clause out of three top-sequents in total, $\llbracket(p \vee$ $\neg p) \wedge(\neg p \wedge p) \rrbracket=1 / 3=0 . \overline{3}$. Stability intervenes to guarantee that any other possible $\overline{\overline{\mathrm{G}} 4}$-derivation of $\Rightarrow(p \vee \neg p) \wedge(\neg p \wedge p)$ will always return the value $0 . \overline{3}$.

In this paper, we aim at expanding this fractional account to modal logic by designing a modal proof-system constrained by bilateralism, invertibility, and stability.

1 As remarked by a referee, there is an important distinction to spot between our system (where a formula may be invalid without its negation being provable) and the more traditional kind of bilateralism where there is a close relation between refutation and proof of negation, and not all formulas are either provable or refutable (e.g., atomic formulas). Although other terms in the literature (for example, "hybrid" in $[13,14]$ ) can describe our system, we prefer to use "bilateralism" as an umbrella term capable of including our system as well. 
Notoriously, the inadequacy of a truth-functional approach to modal logic was established by Dugundji's celebrated result in 1940, according to which Lewis systems from $S 1$ to $S 5$ cannot be semantically characterized by an $n$-valued semantics $[8,9]$. Still, many well-known modal systems such as $K, T, 4, B, G L$, and $S 1-5$ are decidable and satisfy the finite model property [7]. Furthermore, in the past two decades many of the above systems have received a satisfactory proof-theoretic treatment and for each of them different frameworks of proof systems have been furnished.

Initially this treatment attained cut-free and decidable sequent calculi, but failed to encompass modal systems such as $B$ or $S 5$, where the axiom corresponding to symmetry proved to be very hard to handle. The picture changed when suitable generalizations of the classical sequent calculus entered the scene. These new frameworks were presented in two different fashions: either via the internalization of semantics [22, 31], or by enriching the structure of sequents [3, 6, 26]. Both these approaches allowed for the formulation of proof-systems with good structural properties and, in most cases, yielding well-defined decision procedures. In sum, the quest for a well-behaved Gentzen-style proof theory has progressively brought modal logics closer to classical logic by recovering proof-theoretic properties such as symmetry and harmony which typically characterize sequent calculi for classical logic.

In what follows we will be concerned with the normal modal logic $K$, that is the minimal modal system including the $K$-axiom $\square(A \rightarrow B) \rightarrow(\square A \rightarrow \square B)$ and closed under the rule of necessitation: if $A$ is a theorem, so is $\square A$. $K$ represents a base for the other modal systems - obtained via the addition of specific axioms - in which the modality gains more and more sophisticated interpretations. Moreover, our perspective also involves an attempt to develop a proof-theoretic semantics for modal logic by building a bridge between modal and classical logic. Indeed, we subscribe to the claim that proof-theoretic semantics is "seriously incomplete" without an account of the meanings of modal operators in terms of rules of inference [18, p. 724]. There is, however, an important methodological difference between the approach here proposed and the more standard ones [11, 25, 32]. Traditional stances share the view that the meaning of logical operators should be transmitted by the rules governing their use. This is an essentially local approach in the sense that single inference steps are taken to convey an operational interpretation irrespective of the specific formal proof in which inferences occur. In the context of fractional semantics, by contrast, the approach we are pursuing can be classified as global: it appeals, indeed, to the macro-structure of proofs determining the decomposition of formulas and, consequently, to the multiset of clauses displayed as top-sequents. Single inferences still contribute to meaning but, so we argue, the latter can be fully extracted only from the proof as an organic unity.

On the other hand, fractional semantics affords an informational refinement of modal logic, in which, like in classical logic, the notion of validity is traditionally interpreted as a sharp one, without some degree of truth or validity. It is important to appreciate, however, that analyticity and invertibility of the calculus weed out any suspicion that such an informational refinement is an arbitrary one. Indeed, the decomposition induced by the rules of the calculus reduces the validity (or invalidity) of the end-sequent to the validity (or invalidity) of the top-sequents. So, every branch of a derivation terminating in a non-axiomatic sequent may be understood as a successful attempt at falsifying the end-sequent. Therefore, the ratio between axiomatic and complementary sequents determines the degree of validity of a formula. In particular, 
this refinement enables us to propose a novel proof of Dugundji's theorem, relative to $K$, which gets rid of the construction of an infinite countermodel.

Our proof-theoretic machinery comes as a bilateral hypersequent calculus for $K$; as far as we know, this is the first calculus of this type for some systems of modal logic in the literature. Moreover, as well as being a deductive setting in which fractional semantics can be implemented, this system has an intrinsic proof-theoretic interest to the extent that it reconciles the invertibility of the rules (thus eliminating the need for backtracking) with the finiteness of the proof-search space. As a matter of fact, the standard sequent calculus for modal logic $K$ [33] satisfies termination, but it essentially requires a backtracking procedure due to the failure of invertibility. Vice versa, extensions of the standard framework of sequent calculus such as labeled sequent calculi [22], nested sequents [6], or display calculi [34] enjoy full invertibility, but the proof-search space is not finite.

The plan of the paper is as follows. In Section 2, we first introduce the proof-system $\overline{\overline{\mathrm{HK}}}$, a bilateral hypersequent calculus sound and complete with respect to the set of $K$ valid formulas. $\overline{\overline{\mathrm{HK}}}$ is shown to satisfy some desirable structural properties, including the invertibility of the logical rules and cut-elimination. In Section 3, we establish stability and, contextually, we show how to employ $\overline{\overline{\mathrm{HK}}}$ as an algorithm to fractionally evaluate $K$-formulas. Then, in Section 4, such a fractional interpretation becomes a lens through which we closer look at Dugundji's theorem. Finally, Section 5 offers some concluding remarks.

\section{§2. An invertible bilateral hypersequent calculus for $K$.}

2.1. The calculus $\overline{\overline{\mathrm{HK}}}$. We shall be mainly working with hypersequents, introduced under a different name by Mints in the early seventies of the last century [20,21] and independently by Pottinger [27], then further elaborated (and so named) by Avron [1-3]. They are a generalization of the standard notion of sequent in the style of Gentzen. A sequent is a syntactic entity of the form $\Gamma \Rightarrow \Delta$, where $\Gamma, \Delta$ are finite multisets of modal formulas from the set $\mathcal{F}$ recursively defined by the grammar:

$$
\mathscr{F}::=A T|\neg \mathcal{F}| \mathcal{F} \rightarrow \mathcal{F}|\mathcal{F} \wedge \mathcal{F}| \mathcal{F} \vee \mathcal{F} \mid \square \mathcal{F}
$$

with $A T$ collecting the atomic sentences. As usual, $\diamond A$ abridges the formula $\neg \square \neg A$. If $\Gamma=\left[A_{1}, A_{2}, \ldots, A_{n}\right]$, then $\wedge \Gamma$ and $\bigvee \Gamma$ are the two formulas $A_{1} \wedge A_{2} \wedge \cdots \wedge A_{n}$ and $A_{1} \vee A_{2} \vee \cdots \vee A_{n}$, respectively. If $\Gamma=\varnothing$, then we set $\bigwedge \Gamma=\top$ and $\bigvee \Gamma=\perp$, where $\top$ and $\perp$ stand for an arbitrarily selected tautology and contradiction, respectively. With $\square \Gamma$ we mean the multiset $\left[\square A_{1}, \square A_{2}, \ldots, \square A_{n}\right.$ ]. For every formula $A$ we denote with $A^{n}$ the multiset containing exactly $n$ occurrences of $A$.

In general, if $M$ and $N$ are two multisets, we indicate with $M \uplus N$ and \#M their multiset union and $M$ 's cardinality, respectively. A hypersequent, denoted by $\mathcal{G}, \mathcal{H}, \ldots$, is defined as a finite (possibly empty) multiset of sequents written as follows:

$$
\Gamma_{1} \Rightarrow \Delta_{1}\left|\Gamma_{2} \Rightarrow \Delta_{2}\right| \cdots \mid \Gamma_{n} \Rightarrow \Delta_{n}
$$

We shall keep calling "sequents" those hypersequents listing exactly one sequent. The set collecting hypersequents is here indicated with $\mathcal{H}$. Informally speaking, a hypersequent $\mathcal{G}$ proves valid whenever at least one of the sequents listed in $\mathcal{G}$ is valid. 
axioms

$$
\begin{aligned}
& \overline{1} \square \Pi_{1}, \Gamma_{1}, \mathrm{p} \Rightarrow \Delta_{1}, \mathrm{p}\left|\square \Pi_{2}, \Gamma_{2} \Rightarrow \Delta_{2}\right| \ldots \mid \square \Pi_{n}, \Gamma_{n} \Rightarrow \Delta_{n} \\
& \frac{10 \square \Pi_{1}, \Gamma_{1} \Rightarrow \Delta_{1}|\ldots| \square \Pi_{n}, \Gamma_{n} \Rightarrow \Delta_{n}}{\operatorname{ax}} \quad \text { where } \Gamma_{i} \cap \Delta_{i}=\varnothing \text { for all } 1 \leqslant \mathrm{i} \leqslant \mathrm{n}
\end{aligned}
$$

logical rules

$$
\begin{aligned}
& \frac{\vdash^{i} \mathcal{G} \mid \Gamma \Rightarrow \Delta, A}{\left.\right|^{i} \mathcal{G} \mid \Gamma, \neg A \Rightarrow \Delta} \neg \Rightarrow \quad \frac{\vdash^{i} \mathcal{G} \mid A, \Gamma \Rightarrow \Delta}{\left.\right|^{i} \mid \Gamma \Rightarrow \Delta, \neg A} \Rightarrow \neg \\
& \frac{\vdash^{i} \mathcal{G} \mid \Gamma, A, B \Rightarrow \Delta}{\vdash^{i} \mathcal{G} \mid \Gamma, A \wedge B \Rightarrow \Delta} \wedge \Rightarrow \quad \frac{\mathfrak{i}^{i} \mathcal{G}\left|\Gamma \Rightarrow \Delta, A \quad \vdash^{j} \mathcal{G}\right| \Gamma \Rightarrow \Delta, B}{\left.\right|^{i \cdot j} \mathcal{G} \mid \Gamma \Rightarrow \Delta, A \wedge B} \Rightarrow \wedge \\
& \frac{\mathfrak{i}^{i} \mathcal{G}\left|\Gamma, A \Rightarrow \Delta \quad \vdash^{j} \mathcal{G}\right| \Gamma, B \Rightarrow \Delta}{\vdash^{i \cdot j} \mathcal{G} \mid \Gamma, A \vee B \Rightarrow \Delta} \vee \quad \frac{\left.\right|^{i} \mathcal{G} \mid \Gamma \Rightarrow \Delta, A, B}{||^{i} \mathcal{G} \mid \Gamma \Rightarrow \Delta, A \vee B} \Rightarrow \vee \\
& \frac{\mathfrak{\vdash}^{i}\left|\Gamma \Rightarrow \Delta, A \quad \vdash^{j} \mathcal{G}\right| \Gamma, B \Rightarrow \Delta}{\vdash^{i \cdot j} \mathcal{G} \mid \Gamma, A \rightarrow B \Rightarrow \Delta} \rightarrow \quad \frac{\vdash^{i} \mathcal{G} \mid \Gamma, A \Rightarrow \Delta, B}{\left|{ }^{i} \mathcal{G}\right| \Gamma \Rightarrow \Delta, A \rightarrow B} \Rightarrow \rightarrow
\end{aligned}
$$

modal operator rule

$$
\frac{\stackrel{\mid i}{ } \mathcal{G}|\Gamma \Rightarrow A| \square \Gamma, \Gamma^{\prime} \Rightarrow \square \Delta, \Delta^{\prime}}{\left.\right|^{i} \mathcal{G} \mid \square \Gamma, \Gamma^{\prime} \Rightarrow \square A, \square \Delta, \Delta^{\prime}} \square, \text { where } \Gamma^{\prime} \uplus \Delta^{\prime} \subseteq A T
$$

Fig. 1. The $\overline{\overline{\mathrm{HK}}}$ sequent calculus (read $\left.\right|^{1}$ as $\vdash$, and $\vdash^{0}$ as $\left.\dashv\right)$.

Here the meaning of the term "valid" has to be specified depending on the logical context (cf. Definition 3).

The following definition introduces the notion of hyperclause which extends that of clause for standard sequents of classical logic.

Definition 1 (Hyperclauses). A hyperclause is a hypersequent

$$
\square \Pi_{1}, \Gamma_{1} \Rightarrow \Delta_{1}|\cdots| \square \Pi_{n}, \Gamma_{n} \Rightarrow \Delta_{n}
$$

such that $\Gamma_{i} \uplus \Delta_{i} \subset A T$, for all $1 \leqslant i \leqslant n$. An identity hyperclause is such that, for some $i, \Gamma_{i} \uplus \Delta_{i} \neq \varnothing$; otherwise, it is complementary.

EXAMPLE 2.1. An identity hyperclause and a complementary hyperclause, respectively:

$$
p \Rightarrow p|\square(\square p \rightarrow p) \Rightarrow \quad \Rightarrow p| \Rightarrow p \mid \square(\square p \rightarrow p) \Rightarrow
$$

Figure 1 presents the bilateral hypersequent calculus $\overline{\overline{\mathrm{HK}}}$. The rules of $\overline{\overline{\mathrm{HK}}}$ operate on hypersequents signed with the symbols " $\vdash$ " and " $\dashv$ ": we write $\vdash \mathcal{G}$ and $\dashv \mathcal{G}$ to 


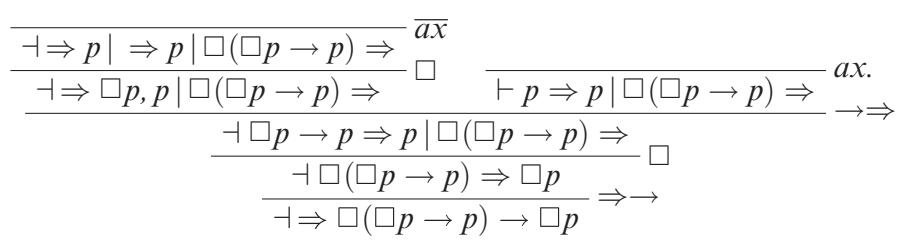

Fig. 2. An example of $\overline{\overline{\mathrm{HK}}}$ proof.

assert the validity and invalidity of $\mathcal{G}$, respectively (cf. Definition 3). For the sake of a more compact notation, in Figure 1 the $\overline{\overline{\mathrm{HK}}}$ rules are expressed by writing 1 and $f^{0}$ to indicate the two signs " $\vdash$ " and " $\dashv$, ," respectively. The calculus is equipped with two axiom rules: the $a x$-rule introduces any identity hyperclause, whilst the $\overline{a x}$-rule introduces whatsoever complementary hyperclause.

From now on, we will indicate derivations with small Greek letters $\pi, \rho, \ldots$. The height $h(\pi)$ of a derivation $\pi$ is given by the number of hypersequents figuring in one of its longest branches. Moreover, we indicate with top $(\pi)$ the multiset of $\pi$ 's top-hypersequents.

EXAMPLE 2.2. Figure 2 displays an $\overline{\overline{\mathrm{HK}}}$-derivation ending in $\dashv \Rightarrow \square(\square p \rightarrow p) \rightarrow \square p$.

REMARK 1. The rule $\square$ is the only rule in which the hypersequent structure comes effectively into play. Informally speaking, it allows us to separate a classical and a modal part in a derivation. In fact, every time this rule is applied, a new hypersequent component is added, thus starting a parallel derivation.

Furthermore, notice that the side condition on the $\square$-rule about the two contexts $\Gamma^{\prime}$ and $\Delta^{\prime}$ is crucial to avoid pathological situations in which, for some hypersequent $\mathcal{G}$, $\overline{\overline{\mathrm{HK}}}$ proves both $\vdash \mathcal{G}$ and $\dashv \mathcal{G}$ as the following:

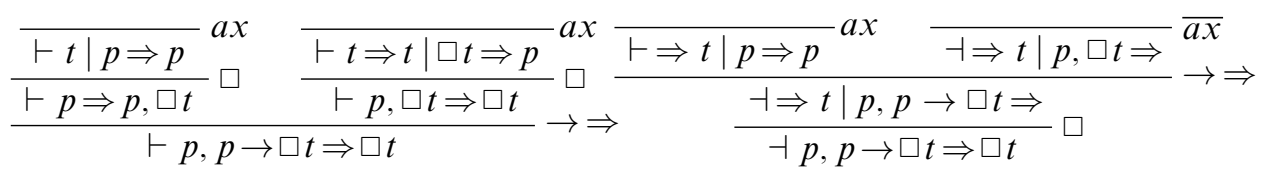

Lemma 2.1. For any hypersequent $\mathcal{G}, \overline{\overline{\mathrm{HK}}}$ proves $\vdash \mathcal{G}$ or it proves $\dashv \mathcal{G}$.

Proof. Any hypersequent which is not a hyperclause can be further analyzed via some suitable bottom-up applications of the rules in which the part concerning the standard/reversed turnstile is simply ignored. Once the decomposition is fully accomplished, we can recover the information about turnstiles by propagating it from the axiom-leaves to the conclusion, according to the rules listed in Figure 1.

2.2. Bilateral soundness and completeness. Let us begin by briefly recalling some basic semantic notions.

Definition 2 ( $K$-model, $K$-validity). A $K$-model $\mathcal{M}$ consists of a triple $\langle W, R, v\rangle$ where $W \neq \varnothing, R \subseteq W^{2}$ and $v: A T \rightarrow \mathcal{P}(W)$ is a valuation function. For every $w \in W$ and every formula $A$, the relation $w \Vdash A$ is thus defined:

- $w \Vdash p$ iff $w \in v(p)$ for every $p \in A T$,

- $w \Vdash \neg B$ iff $w \nVdash B$,

- $w \Vdash B \wedge C$ iff $w \Vdash B$ and $w \Vdash C$, 
- $w \Vdash B \vee C$ iff $w \Vdash B$ or $w \Vdash C$,

- $w \Vdash B \rightarrow C$ iff if $w \nVdash B$ or $w \Vdash C$,

- $\quad w \Vdash \square B$ iff for every $w^{\prime}$, if $R w w^{\prime}$, then $w^{\prime} \vDash B$.

The sentence $A$ is true in $\mathcal{M}$ iff $w \Vdash A$, for all $w \in W$. Moreover, $A$ is valid, in symbols $\vDash A$, in case it is true in any $K$-model; otherwise, it is invalid.

According to the customary operational interpretation of sequents, $\Gamma \Rightarrow \Delta$ is $K$ valid if the formula $\wedge \Gamma \rightarrow \bigvee \Delta$ is $K$-valid. The next definition accommodates the notion of $K$-validity to hypersequents.

Definition 3 ( $K$-valid hypersequents). For any hypersequent $\mathcal{G} \equiv \Gamma_{1} \Rightarrow \Delta_{1}|\cdots| \Gamma_{n} \Rightarrow$ $\Delta_{n}$, the function hfor: $\mathscr{H} \mapsto \mathcal{F}$ is defined as follows:

$$
\mathrm{hfor}[\mathcal{G}]=\square\left(\bigwedge \Gamma_{1} \rightarrow \bigvee \Delta_{1}\right) \vee \cdots \vee \square\left(\bigwedge \Gamma_{n} \rightarrow \bigvee \Delta_{n}\right)
$$

A hypersequent $\mathcal{G}$ is said to be $K$-valid just in case the formula $\mathrm{hfor}$ [G] is $K$-valid.

EXAmPLE 2.3. Under Definition 3, the hypersequent $p \Rightarrow p|p \Rightarrow q| \square(p \vee q) \Rightarrow$ turns out to be $K$-valid, being valid the following formula:

$$
\mathrm{hfor}[p \Rightarrow p|p \Rightarrow q| \square(p \vee q) \Rightarrow] \equiv \square(p \rightarrow p) \vee \square(p \rightarrow q) \vee \square(\square(p \vee q) \rightarrow \perp) \text {. }
$$

It should not escape notice that hypersequents cannot be treated naively by interpreting $\Gamma_{1} \Rightarrow \Delta_{1}|\cdots| \Gamma_{n} \Rightarrow \Delta_{n}$ as the formula $\left(\bigwedge \Gamma_{1} \rightarrow \vee \Delta_{1}\right) \vee \cdots \vee\left(\bigwedge \Gamma_{n} \rightarrow\right.$ $\left.\bigvee \Delta_{n}\right)$. Such an interpretation would make collapse, for instance, the two hypersequents $\Rightarrow A \vee \neg A$ and $\Rightarrow A \mid A \Rightarrow$ into the same formula $A \vee \neg A$.

Lemma 2.2. Any identity (resp. complementary) hyperclause is K-valid (resp. not K-valid).

Proof. Consider a generic identity hyperclause $\mathcal{G} \mid \Gamma, p \Rightarrow \Delta, p$. Then, the formula $\square(\bigwedge \Gamma \wedge p \rightarrow \bigvee \Delta \vee p)$ is clearly $K$-valid, and so is the whole formula $\mathrm{hfor}[\mathcal{G}] \vee$ $\square(\wedge \Gamma \wedge p \rightarrow \bigvee \Delta \vee p)$

Consider now a complementary hyperclause $\square \Pi_{1}, \Gamma_{1} \Rightarrow \Delta_{1}|\cdots| \square \Pi_{n}, \Gamma_{n} \Rightarrow \Delta_{n}$. For each sequent $\square \Pi_{i}, \Gamma_{i} \Rightarrow \Delta_{i}$, take the trivial $K$-model $\mathcal{M}_{i}=\left\langle\left\{w_{i}\right\}, \varnothing, v_{i}\right\rangle$ where the interpretation function $v_{i}$ is thus defined:

- $v_{i}(p)=\left\{w_{i}\right\}$, for all $p \in \Gamma_{i}$,

- $v_{i}(q)=\varnothing$, for any atom $q \notin \Gamma_{i}$.

For every $i, \mathcal{M}_{i}, w_{i} \Vdash \bigwedge \square \Pi_{i}$ (this is vacuously true by Definition 2) and $\mathcal{M}_{i}, w_{i} \Vdash \bigwedge \Gamma_{i}$, but $\mathcal{M}_{i}, w_{i} \nVdash \bigvee \Delta_{i}$. Therefore, $\mathcal{M}_{i}, w_{i} \nVdash\left(\bigwedge \square \Pi_{i} \wedge \wedge \Gamma_{i}\right) \rightarrow \bigvee \Delta_{i}$. Then, we can easily exhibit the sought countermodel by considering

$$
\mathcal{M}=\left\langle\left\{w_{0}, w_{1}, \ldots, w_{n}\right\},\left\{\left(w_{0}, w_{i}\right) \mid 1 \leqslant i \leqslant n\right\}, \bigcup_{1 \leqslant i \leqslant n} v_{i}\right\rangle .
$$

Indeed, for every $i$, it turns out that $R w_{0} w_{i}$ and $\mathcal{M}, w_{i} \nVdash\left(\bigwedge \square \Pi_{i} \wedge \wedge \Gamma_{i}\right) \rightarrow \bigvee \Delta_{i}$, so that we can conclude $\mathcal{M}, w_{0} \nVdash$ hfor $\left[\square \Pi_{1}, \Gamma_{1} \Rightarrow \Delta_{1}|\cdots| \square \Pi_{n}, \Gamma_{n} \Rightarrow \Delta_{n}\right]$.

Lemma 2.3 (Modal disjunction property). If $\vDash \square A \vee \square B$, then $\vDash A$ or $\vDash B$.

Proof. The reader is referred to [7, pp. 90-91]. 
THEOREM 2.4 (Soundness). If the system $\overline{\overline{\mathrm{HK}}}$ proves $\vdash \mathcal{G}$ and $\dashv \mathcal{H}$, then the hypersequents $\mathcal{G}$ and $\mathcal{H}$ are $K$-valid and $K$-invalid, respectively.

Proof. The proof is carried out by induction on the height of a generic $\overline{\overline{\mathrm{HK}}}$-proof $\pi$. Lemma 2.2 immediately supplies the base case. As for the inductive case, we limit ourselves to discussing the case in which $\pi$ 's last inference step is an application of the $\square$-rule. We distinguish two cases according to whether $\pi$ ends in $\vdash \mathcal{G}$ or $\dashv \mathcal{H}$.

- $(\vdash \mathcal{G})$. By inductive hypothesis, it results that the premise $\mathcal{G}^{\prime}|\Gamma \Rightarrow A| \square \Gamma, \Gamma^{\prime} \Rightarrow$ $\square \Delta, \Delta^{\prime}$ is $K$-valid, where $\Gamma^{\prime}, \Delta^{\prime} \subset A T$. In case $\mathrm{hfor}\left[\mathcal{G}^{\prime}\right]$ is a $K$-valid formula, we immediately get the desired conclusion. Otherwise, by Lemma 2.3 , at least one of the two formulas $\bigwedge \Gamma \rightarrow A$ and $\left(\bigwedge \square \Gamma \wedge \wedge \Gamma^{\prime}\right) \rightarrow\left(\bigvee \square \Delta \vee \bigvee \Delta^{\prime}\right)$ turns out to be $K$-valid: in both cases the conclusion easily follows from the monotonicity of $\square$.

- $(\dashv \mathcal{H})$. According to our inductive hypothesis, the hypersequent $\mathcal{G} \mid \Gamma \Rightarrow$ $A \mid \square \Gamma, \Gamma^{\prime} \Rightarrow \square \Delta, \Delta^{\prime}$ (with $\Gamma^{\prime}, \Delta^{\prime} \subset A T$ and $\Gamma^{\prime} \cap \Delta^{\prime}=\varnothing$ ) is now taken to be $K$-invalid. Consider the hypersequent $\mathcal{G} \equiv \Pi_{1} \Rightarrow \Sigma_{1}|\cdots| \Pi_{n} \Rightarrow \Sigma_{n}$ and let $i$ and $j$ be two parameters ranging over the sets $\{1, \ldots, n\}$ and $\{1, \ldots, n+2\}$, respectively. Clearly, none of the formulas $\wedge \Pi_{i} \rightarrow \bigvee \Delta_{i}$ is $K$-valid. The two formulas, $\bigwedge \square \Gamma \wedge \wedge \Gamma^{\prime} \rightarrow \bigvee \square \Delta \vee \vee \Delta^{\prime}$ and $\bigwedge \Gamma \rightarrow A$, are not $K$-valid as well. This means that there is a family of models $\mathcal{M}_{j}=\left\langle W_{j}, R_{j}, v_{j}\right\rangle$ such that:

(1) $\left\langle W_{j}, R_{j}\right\rangle$ is a irreflexive, asymmetric, and intransitive tree with root $w_{j}$ [5, p. 218],

(2) $\mathcal{M}_{i}, w_{i} \Vdash \wedge \Pi_{i}$ and $\mathcal{M}_{i}, w_{i} \nVdash \bigvee \Sigma_{i}$,

(3) $\mathcal{M}_{n+1}, w_{n+1} \Vdash \square \wedge \Gamma \wedge \wedge \Gamma^{\prime}$ and $\mathcal{M}_{i}, w_{n+1} \nVdash \bigvee \square \Delta \vee \bigvee \Delta^{\prime}$,

(4) $\mathcal{M}_{n+2}, w_{n+2} \Vdash \Lambda \Gamma$ and $\mathcal{M}_{n+2}, w_{n+2} \nVdash A$.

Thus, finally consider the model

$$
\begin{gathered}
\mathcal{M}=\left\langle\bigcup_{j=1}^{n+2} w_{j} \cup\left\{w_{0}\right\}, \bigcup_{j=1}^{n+2} R_{j} \cup\left\{\left(w_{n+1}, w_{n+2}\right)\right\}\right. \\
\left.\cup\left\{\left(w_{0}, w_{k}\right) \mid 1 \leqslant k \leqslant n+1\right\}, \bigcup_{j=1}^{n+2} v_{j}\right\rangle
\end{gathered}
$$

and observe that $\mathcal{M}, w_{0} \nVdash \mathrm{hfor}\left[\mathcal{G} \mid \square \Gamma, \Gamma^{\prime} \Rightarrow \square \Delta, \Delta^{\prime}, \square A\right]$ which yields the desired conclusion. (Let us observe that the constraint $\Gamma^{\prime}, \Delta^{\prime} \subset A T$ is essential in the final part of the previous argument to prevent that $\mathcal{M}, x_{n+1} \nVdash \wedge \Gamma^{\prime}$ by adding $R\left(w_{n+1}, w_{n+2}\right)$ to the model $)$.

COROLlary 2.5 (Completeness). If the hypersequent $\mathcal{G}$ is $K$-valid, then $\overline{\overline{\mathrm{HK}}}$ proves $\vdash \mathcal{G}$. Otherwise, $\overline{\overline{\mathrm{HK}}}$ proves $\dashv \mathcal{G}$.

Proof. Assume that $\mathcal{G}$ is $K$-valid, but $\overline{\overline{\mathrm{HK}}}$ does not prove $\vdash \mathcal{G}$. By Lemma 2.1, $\overline{\overline{\mathrm{HK}}}$ proves $\dashv \mathcal{G}$ and so, by Theorem 2.4, $\mathcal{G}$ would be $K$-invalid. Therefore, $\overline{\overline{\mathrm{HK}}}$ does prove $\vdash \mathcal{G}$. The case in which $\mathcal{G}$ is $K$-invalid can be treated symmetrically.

Let us call HK the hypersequent system obtained from $\overline{\overline{\mathrm{HK}}}$ by dropping the complementary axiom and removing everywhere the signs " - " and " $\dashv$." What we 
get in this way is a complete "monolateral" characterization of the system $K$. More formally:

THeOREM 2.6. HK proves $\Rightarrow A$ if and only if $\vDash A$.

Proof. Any proof in which no application of the complementary axiom occurs will necessarily end with a hypersequent signed with " $\vdash$." Then, by Theorem 2.4 , the formula $\square A$ is valid and, by the rule of denecessitation (from $\vDash \square A$ to $\vDash A$ ), we finally get the claim of the theorem.

REMARK 2. The rule of denecessitation is indeed sound in $K$, but it does not prove admissible in every modal system, for example $K 5$. Therefore, in order to extend the fractional interpretation to such systems a new strategy would be required.

Corollary 2.7. The bilateral cut rule:

$$
\frac{\vdash^{i} \mathcal{G}|\Gamma \Rightarrow \Delta, A \quad|^{j} \mathcal{G} \mid A, \Gamma \Rightarrow \Delta}{\left.\right|^{i \cdot j} \mathcal{G} \mid \Gamma \Rightarrow \Delta}
$$

is admissible in $\overline{\overline{\mathrm{HK}}}$.

Proof. We observe that the above-mentioned bilateral rendition of the cut-rule proves sound in $\overline{\overline{\mathrm{HK}}}$. By applying Corollary 2.5 we finally get the claim of the theorem.

In the proof of the next lemma, we will need to employ the notion of principal formula of an inference rule. In case of inference rules introducing one of the classical connectives, the notion of principal formula is just the familiar one (cf. [30]). As regards to the rule for $\square$ in Figure 1, we take $\square A$ to be the principal formula.

THEOREM 2.8 (Invertibility). Each $\overline{\overline{\mathrm{HK}}}$ logical rule proves height-bounded invertible with preservation of top-hypersequents, namely:

(i) for any instance of a unary rule $\frac{{\frac{i}{i} \mathcal{G}_{1}}^{i} \mathcal{G}}{\mathfrak{G}^{\prime}}$, if $\overline{\overline{\mathrm{HK}}}$ proves $\vdash^{i} \mathcal{G}$ by way of a proof $\pi$, then it also proves $1^{i} \mathcal{G}_{1}$ by way of a proof $\pi_{1}$ such that $h\left(\pi_{1}\right) \leqslant h(\pi)$ and $\operatorname{top}\left(\pi_{1}\right)=\operatorname{top}(\pi)$;

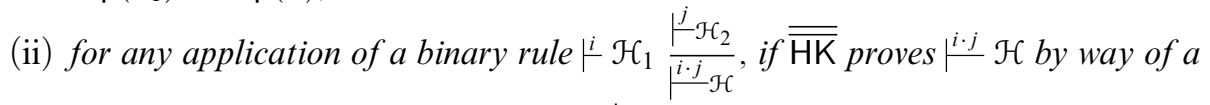
proof $\pi$, then it also proves $\vdash^{i} \mathcal{G}_{1}$ and $\left.\right|^{j} \mathcal{G}_{2}$ by way of two proofs $\pi_{1}$ and $\pi_{2}$ such that $h\left(\pi_{1}\right), h\left(\pi_{2}\right) \leqslant h(\pi)$ and $\operatorname{top}\left(\pi_{1}\right) \uplus \operatorname{top}\left(\pi_{2}\right)=\operatorname{top}(\pi)$.

Proof. We distinguish the cases according to the rule instances. For every rule instance we proceed by induction on the height $h(\pi)$ of the derivation $\pi$ leading to the end-hypersequent under consideration, and by distinguishing subcases on the basis of the last rule applied. We deal with two rules; the other ones can be treated likewise.

- $(\vee \Rightarrow)$ For $h(\pi)=1$, the claim holds vacuously. If $h(\pi)>1$ and the principal formula of $\pi$ 's last rule occurs in the context of the end-hypersequent, then the conclusion follows by applying the inductive hypothesis to the premise(s) and then the same rule again. If $h(\pi)>1$ and the last rule applied acted in the component that we are considering, then it cannot be a $\square$-rule due to context 
restrictions. We limit ourselves to treat the case in which the last inference is a $(\Rightarrow \wedge)$-rule.

$$
\begin{aligned}
& \pi_{1} \quad \vdots \pi_{2} \\
& \frac{\vdash^{i} \mathcal{G}|A \vee B, \Gamma \Rightarrow \Delta, C \quad|^{j} \mathcal{G} \mid A \vee B, \Gamma \Rightarrow \Delta, D}{\left.\right|^{i \cdot j} \mathcal{G} \mid A \vee B, \Gamma \Rightarrow \Delta, C \wedge D} \Rightarrow \wedge
\end{aligned}
$$

By applying our inductive hypothesis, we obtain the following four derivations:

$$
\begin{aligned}
& \rho_{1}^{\prime}:\left.\quad\right|^{i_{1}} \mathcal{G} \mid A, \Gamma \Rightarrow \Delta, C, \\
& \rho_{1}^{\prime \prime}: \quad i_{2} \mathcal{G} \mid B, \Gamma \Rightarrow \Delta, C \text {, } \\
& \rho_{2}^{\prime}: \quad j_{1}^{j_{1}} \mathcal{G} \mid A, \Gamma \Rightarrow \Delta, D \text {, } \\
& \rho_{2}^{\prime \prime}: \quad f_{2} \mathcal{G} \mid B, \Gamma \Rightarrow \Delta, D,
\end{aligned}
$$

with $i_{1} \cdot i_{2}=i$ and $j_{1} \cdot j_{2}=j$, that we recombine as follows:

$$
\begin{array}{cc}
\vdots \rho_{1}^{\prime} & \vdots \rho_{2}^{\prime} \\
\pi^{\prime}: \frac{\left.\right|^{i_{1}} \mathcal{G} \mid A, \Gamma \Rightarrow \Delta, C}{\left.\right|^{i_{1} \cdot j_{1}}} \mathcal{G} \mid A, \Gamma \Rightarrow \Delta, C \wedge D & \left.\right|^{j_{1}} \mathcal{G} \mid A, \Gamma \Rightarrow \Delta, D \\
\vdots \rho_{1}^{\prime \prime} & \vdots \rho_{2}^{\prime \prime} \\
\pi^{\prime \prime}: \frac{\left.\right|^{i_{2}} \mathcal{G} \mid B, \Gamma \Rightarrow \Delta, C}{\left.\right|^{i_{2} \cdot j_{2}}} \mathcal{G} \mid B, \Gamma \Rightarrow \Delta, C \wedge D
\end{array}
$$

with $\left(i_{1} \cdot j_{1}\right) \cdot\left(i_{2} \cdot j_{2}\right)=i \cdot j$. Finally, we observe that $\operatorname{top}\left(\pi_{1}\right)=\operatorname{top}\left(\rho_{1}\right) \uplus$ $\operatorname{top}\left(\rho_{1}^{\prime \prime}\right)$ and $\operatorname{top}\left(\pi_{2}\right)=\operatorname{top}\left(\rho_{2}^{\prime}\right) \uplus \operatorname{top}\left(\rho_{2}^{\prime \prime}\right)$. From these two facts we can easily derive top $(\pi)=\operatorname{top}\left(\pi^{\prime}\right) \uplus \operatorname{top}\left(\pi^{\prime \prime}\right)$.

- ( $\square)$ For $h(\pi)=1$, the claim holds vacuously. If $h(\pi)>1$ and the principal formula occurs in the context of the end-hypersequent, then, as in the previous case, the conclusion follows by applying the inductive hypothesis to the premise(s) and then the same rule again. If $h(\pi)>1$ and the last rule acted in the component under consideration, then it will be a $\square$-rule. If the principal formula of the rule instance coincides with the principal formula of the application of the $\square$-rule, the claim clearly holds. Otherwise, we are in the following situation:

$$
\begin{gathered}
\vdots_{\pi_{1}} \\
\frac{{ }^{i} \mathcal{G}|\Gamma \Rightarrow B| \square \Gamma, \Gamma^{\prime} \Rightarrow \square \Delta^{\prime \prime}, \square A, \Delta^{\prime}}{|i \mathcal{G}| \square \Gamma, \Gamma^{\prime} \Rightarrow \square \Delta^{\prime \prime}, \square A, \square B, \Delta^{\prime}}
\end{gathered}
$$

where, clearly, $\operatorname{top}\left(\pi_{1}\right)=\operatorname{top}(\pi)$. We can now apply our inductive hypothesis so as to get a derivation $\rho$ of $\left.\right|^{i} \mathcal{G}|\Gamma \Rightarrow B| \Gamma \Rightarrow A \mid \square \Gamma, \Gamma^{\prime} \Rightarrow \square \Delta^{\prime \prime}, \Delta^{\prime}$ such that $\operatorname{top}(\rho)=\operatorname{top}\left(\pi_{1}\right)$ and $h(\rho) \leqslant h\left(\pi_{1}\right)$. A final application of the $\square$-rule yields the desired result. 
Proposition 2.9. For any $\overline{\overline{\mathrm{HK}}}$-proof $\pi$ ending in the hypersequent $\mathcal{G}$ and such that $\operatorname{top}(\pi)=\left\{\mathcal{T}_{1}, \mathcal{T}_{2}, \ldots, \mathcal{T}_{n}\right\}$, the two formulas $\mathrm{hfor}[\mathcal{G}]$ and $\bigwedge_{1 \leqslant i \leqslant n} \mathrm{hfor}\left[\mathcal{T}_{i}\right]$ are equivalent, i.e., $\vDash \mathrm{hfor}[\mathcal{G}]$ if, and only if, $\vDash \bigwedge_{1 \leqslant i \leqslant n} \mathrm{hfor}\left[\mathcal{T}_{i}\right]$.

Proof. By the soundness theorem we know that, for each logical rule, the conclusion is $K$-valid just in case the premise(s) is(are) $K$-valid as well. Thus, it immediately follows that $\vDash$ hfor $[\mathcal{G}]$ if, and only if, $\vDash \bigwedge$ hfor $\left[\mathcal{T}_{i}\right]$.

THEOREM 2.10. The following statements hold:

(1) $\overline{\overline{\mathrm{HK}}}$ proves $\vdash^{i} \mathcal{G} \mid \square \Gamma^{\prime}, \Gamma \Rightarrow \Delta$ if, and only if, $\overline{\overline{\mathrm{HK}}}$ proves $\vdash \mathcal{G} \mid \Gamma \Rightarrow \Delta$ with $\Gamma \uplus \Delta \subset A T$.

(2) If $\overline{\overline{\mathrm{HK}}}$ proves $i^{i} \mathcal{G} \mid \square \Gamma^{\prime} \Rightarrow$ with $\mathcal{G} \neq \varnothing$, then $\overline{\overline{\mathrm{HK}}}$ proves $\downarrow^{i} \mathcal{G}$.

Proof. We discuss the two items separately.

(1) We argue by induction on the height of derivations. From right to left the proof is quite straightforward. As for the left-to-right direction, we proceed as follows. If $n=0$, then $\mathcal{G} \mid \square \Gamma^{\prime}, \Gamma \Rightarrow \Delta$ is either an instance of $a x$ or of $\overline{a x}$, and so is $\mathcal{G} \mid \Gamma \Rightarrow \Delta$. If $n>0$ the proof follows by applying the inductive hypothesis to the premises of the last rule of the derivation, as no formula in $\square \Gamma^{\prime}, \Gamma \Rightarrow \Delta$ is principal due to the design of the rules.

(2) If $\overline{\overline{\mathrm{HK}}}$ proves $\stackrel{i}{i}^{\mathcal{G}} \mid \square \Gamma^{\prime} \Rightarrow$, then, by the previous item, it also proves $\left.\right|^{i} \mathcal{G} \mid \Rightarrow$. Moreover, $\overline{\overline{\mathrm{HK}}}$ proves $\left.\right|^{i} \mathcal{G} \mid \Rightarrow$ if and only if $\left.\right|^{i} \mathcal{G}$ is also provable, thus we obtain the desired conclusion.

As a consequence of Proposition 2.9 and Theorem 2.10, the calculus $\overline{\overline{\mathrm{HK}}}$ allows us to reduce the validity (invalidity) of a given end-hypersequent to the validity (invalidity) of the conjunction of axiomatic or complementary hyperclauses which contain only atomic sentences. $^{2}$

\section{§3. Stability and fractional interpretation.}

3.1. The stability theorem. Our concern now is that of establishing that the system $\overline{\overline{\mathrm{HK}}}$ enjoys the stability property, i.e., the fact that any two proofs ending with the same hypersequent always display the same multiset of top-hypersequents. To put it differently, two proofs having the same end-hypersequent may only differ in the specific order in which their logical rules are applied.

THEOREM 3.1 (Stability). If $\pi$ and $\rho$ are two $\overline{\overline{\mathrm{HK}}}$-derivations ending with the same signed hypersequent, then $\operatorname{top}(\pi)=\operatorname{top}(\rho)$.

Proof. We proceed by induction on $h(\pi)$. If $h(\pi)=1$, then $\pi=\rho$, and so top $(\pi)=$ top $(\rho)$. Otherwise, let's distinguish two cases depending on whether $\pi$ 's last rule is unary or binary.

2 Observe that item (2) in the above theorem does not hold for other modal logics, such as $K D$, in which $\neg \square \perp$ is a theorem. 
- First, consider the situation whereby $\pi$ 's last inference is an application of a unary rule and let $\pi_{1}$ indicate $\pi$ 's subproof ending in $\mid i \mathcal{G}$.

$\begin{array}{cc}\pi & \rho \\ \vdots \pi_{1} & \vdots \\ \frac{\vdash^{i} \mathcal{G}_{1}}{\vdash^{i} \mathcal{G}} & \vdash^{i} \mathcal{G}\end{array}$

Clearly, top $\left(\pi_{1}\right)=\operatorname{top}(\pi)$ and $h\left(\pi_{1}\right)<h(\pi)$. By Theorem 2.8, there exists a proof $\rho_{1}$ of $\vdash^{i} \mathcal{G}_{1}$ such that $\operatorname{top}\left(\rho_{1}\right)=\operatorname{top}(\rho)$. By inductive hypothesis, we get $\operatorname{top}\left(\pi_{1}\right)=\operatorname{top}\left(\rho_{1}\right)$ and, lastly, $\operatorname{top}(\pi)=\operatorname{top}(\rho)$.

- Consider now the case in which $\pi$ 's last inference is an application of a binary rule and $\pi_{1}$ and $\pi_{2}$ are the two subproofs of $\pi$ ending in $\vdash^{i} \mathcal{H}_{1}$ and $\vdash^{j} \mathcal{H}_{2}$, respectively.

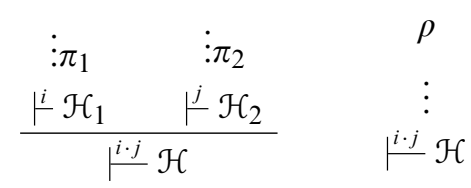

Note that $\operatorname{top}\left(\pi_{1}\right) \uplus \operatorname{top}\left(\pi_{2}\right)=\operatorname{top}(\pi)$ and, clearly, $h\left(\pi_{1}\right), h\left(\pi_{2}\right)<h(\pi)$. Next, we apply Theorem 2.8 to $\rho$ in order to infer the existence of two derivations $\rho_{1}$ and $\rho_{2}$ ending, respectively, in $\vdash^{i} \mathcal{H}_{1}$ and $\vdash^{j} \mathcal{H}_{2}$, and such that $\operatorname{top}\left(\rho_{1}\right) \uplus \operatorname{top}\left(\rho_{2}\right)=\operatorname{top}(\rho)$. By inductive hypothesis, $\operatorname{top}\left(\rho_{1}\right) \uplus \operatorname{top}\left(\rho_{2}\right)=$ $\operatorname{top}\left(\pi_{1}\right) \uplus \operatorname{top}\left(\pi_{2}\right)$, thence we conclude that $\operatorname{top}(\pi)=\operatorname{top}(\rho)$.

3.2. Fractional interpretation. Insofar as $\overline{\overline{\mathrm{HK}}}$ is a bilateral system satisfying stability, any logical formula $A$ univocally determines a specific multiset of hyperclauses, regardless of the particular proof designed to compute such a multiset. This paves the way for the proof-based semantics proposed below.

Definition 4. Given a formula $A, \operatorname{top}(A)$ is the multiset of the top-hyperclauses in any of the $\overline{\overline{\mathrm{HK}}}$-derivations ending in $(\vdash$ or $\dashv) \Rightarrow A$. The multiset top $(A)$ is partitioned into the two multisets $\operatorname{top}^{1}(A)$ and $\operatorname{top}^{0}(A)$ collecting, respectively, all the hyperclauses signed by " $\vdash$ " and the hyperclauses signed by " $\dashv$."

Definition 5. Let $\mathbb{Q}^{*}=[0,1] \cap \mathbb{Q}$, i.e., $\mathbb{Q}^{*}$ is the set of the rational numbers in the closed interval $[0,1]$. The evaluation function $\llbracket \cdot \rrbracket: \mathcal{F} \mapsto \mathbb{Q}^{*}$ is defined as follows: for any logical formula $A, \llbracket A \rrbracket=\frac{\# \operatorname{top}^{1}(A)}{\# \operatorname{top}(A)}$.

EXAMPLE 3.1. Getting back to the derivation $\pi$ proposed in Figure 2, we have:

$$
\begin{aligned}
& \operatorname{top}^{0}(\pi)=\{\Rightarrow p|\Rightarrow p| \square(\square p \rightarrow p) \Rightarrow\}, \\
& \operatorname{top}^{1}(\pi)=\{p \Rightarrow p \mid \square(\square p \rightarrow p) \Rightarrow\} .
\end{aligned}
$$

Under Definition 5, we have $\llbracket \square(\square p \rightarrow p) \rightarrow \square p \rrbracket=0.5$. In effect, any $\overline{\overline{\mathrm{HK}}}$-proof ending in $\Rightarrow \square(\square p \rightarrow p) \rightarrow \square p$ displays one identity axiom out of two axioms in total.

Figure 3 lists some other formulas of $K$ together with their corresponding fractional value. For example, below is the proof of $(\square p \rightarrow \square p) \wedge(\square q \wedge \square r)$ : 


\begin{tabular}{|c|c|}
\hline Formula & Value \\
\hline$\square(p \vee \neg p) \rightarrow(\square p \vee \square \neg p)$ & 0.75 \\
\hline$\square(\square p \rightarrow p) \rightarrow \square p$ & 0.5 \\
\hline$(\square p \rightarrow \square q) \rightarrow \square(p \rightarrow q)$ & 0.5 \\
\hline$\square(p \rightarrow \diamond q) \wedge(\diamond p \rightarrow \diamond q)$ & 0.5 \\
\hline$(\square p \rightarrow \square p) \wedge(\square q \wedge \square r)$ & $0 . \overline{3}$ \\
\hline$((p \wedge q) \wedge r) \wedge \square(s \vee \neg s)$ & 0.25 \\
\hline
\end{tabular}

Fig. 3. Some modal formulas together with their fractional value.

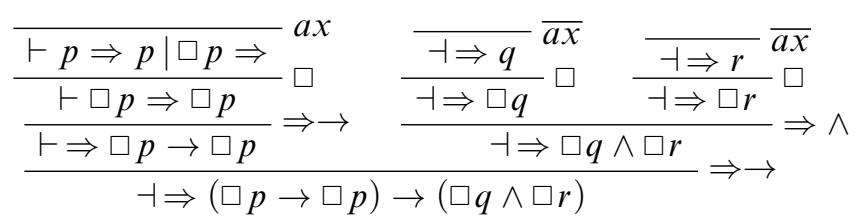

As the proof displays one identity axiom out of three axiom applications in total, we get $\llbracket(\square p \rightarrow \square p) \wedge(\square q \wedge \square r) \rrbracket=0 . \overline{3}$.

Theorem 3.2 (Conservativity). For any formula $A, \llbracket A \rrbracket=1$ if, and only if, $\vDash A$.

Proof. By Theorem 2.4, any formula $A$ is $K$-valid just in case $\operatorname{top}^{1}(A)=\operatorname{top}(A)$.

Notoriously, the lack of truth-functionality is an endemic phenomenon when modalities come into play. It should be observed, however, that in a fractional setting truth-functionality fails already at the very classical level; in this regard, easy examples have been presented in [24].

Let $\mathcal{F}^{c}$ be the language of classical propositional logic. The next theorem establishes the surjectivity of the interpretation function $\llbracket$. \. In particular, we have:

Theorem 3.3. For any $q \in \mathbb{Q}^{*}:(i)$ there is a formula $A \in \mathcal{F}^{c}$ s.t. $\llbracket A \rrbracket=q$, and (ii) there is a formula $B \in \mathscr{F}-\mathscr{F}^{c}$ s.t. $\llbracket B \rrbracket=q$.

Proof. Let $q=m / n$, where $m, n \in \mathbb{N}^{+}$and $m \leqslant n$. (i) Consider the formula $\bigwedge(p \vee$ $\neg p)^{m} \wedge \wedge p^{n-m}$. It is immediate to see that $\llbracket \wedge(p \vee \neg p)^{m} \wedge \wedge p^{n-m} \rrbracket={ }^{m} / n=q$.

(ii) We consider now the modal formula $\bigwedge(\square(p \wedge q) \rightarrow \square p)^{m} \wedge \wedge(\square p)^{n-m}$ in $\mathcal{F}-$ $\mathcal{F}^{c}$. It turns out, similarly, that $\llbracket \wedge(\square(p \wedge q) \rightarrow \square p)^{m} \wedge \wedge(\square p)^{n-m} \rrbracket=m / n=q$.

REMARK 3. As corollary of Theorem 3.3 and from the density of $\mathbb{Q}^{*}$, it results that for any pair of modal formulas $A, B$ with $\llbracket A \rrbracket<\llbracket B \rrbracket$, there always exists a formula $C \in \mathcal{F}^{c}$ such that $\llbracket A \rrbracket<\llbracket C \rrbracket<\llbracket B \rrbracket$.

In a sense, the fractional approach illustrates the continuity between modal logic and classical logic. This claim is strengthened by observing that, in general, for every formula $A, \llbracket A \rrbracket=\llbracket \square A \rrbracket$. However, under fractional semantics modal logic is not monotone, unlike classical logic. To witness this fact, consider the formula $\square p \wedge(p \vee$ $\neg p)$, which gets the fractional value $1 / 2$, whereas the formula $\neg \square(q \vee r) \vee(\square p \wedge(p \vee$ $\neg p)$ ) receives the value $1 / 3$. Nevertheless, a partial result on monotonicity can be recovered if we restrict to classical formulas. 
Lemma 3.4. Let $\pi$ be a derivation in $\overline{\overline{\mathrm{HK}}}$ of the hypersequent $\left.\right|^{i} \mathcal{G} \mid \Gamma \Rightarrow \Delta$. For any multiset $\Pi, \Sigma \subset A T$, there is a derivation $\pi^{\prime}$ of $\vdash^{j} \mathcal{G} \mid \Pi, \Gamma \Rightarrow \Delta, \Sigma$ such that $j \geqslant i$, $\# \operatorname{top}(\pi)=\# \operatorname{top}\left(\pi^{\prime}\right)$ and \#top ${ }^{1}\left(\pi^{\prime}\right) \geqslant \operatorname{top}^{1}(\pi)$.

Proof. The proof is led by induction on $h(\pi)$.

If $h(\pi)=0$, then $\vdash^{i} \mathcal{G} \mid \Gamma \Rightarrow \Delta$ is clearly an instance of an axiom. In particular, when $i=1$, we have \#top ${ }^{1}\left(\pi^{\prime}\right)=\# \operatorname{top}^{1}(\pi)$; otherwise, \#top ${ }^{1}\left(\pi^{\prime}\right) \geqslant \# \operatorname{top}^{1}(\pi)$, as the addition of $\Pi, \Sigma$ may turn a complementary hyperclause into an identity one.

If $h(\pi)>0$, we proceed by cases considering $\pi$ 's last rule. We detail here just the case in which the last rule is an application of the $\square$-rule. There are two further subcases to be distinguished depending on whether the $\square$-rule directly involves the sequent $\Gamma \Rightarrow \Delta$ or some other sequent-components in the hypersequent under consideration. In the second case, the claim follows by inductive hypothesis and an application of $\square$. In the first, we have $\Gamma \Rightarrow \Delta \equiv \square \Gamma^{\prime}, \Gamma^{\prime \prime} \Rightarrow \square \Delta^{\prime}, \Delta^{\prime \prime}, \square A$, with $\Gamma^{\prime \prime} \uplus \Delta^{\prime \prime} \subseteq A T$ and:

$$
\frac{\vdash^{i} \mathcal{G}\left|\Gamma^{\prime} \Rightarrow A\right| \square \Gamma^{\prime}, \Gamma^{\prime \prime} \Rightarrow \square \Delta^{\prime}, \Delta^{\prime \prime}}{\vdash^{i} \mathcal{G} \mid \square \Gamma^{\prime}, \Gamma^{\prime \prime} \Rightarrow \square \Delta^{\prime}, \Delta^{\prime \prime}, \square A} \square
$$

By inductive hypothesis we obtain a derivation $\rho$ of $\left.\right|^{j} \mathcal{G}\left|\Gamma^{\prime} \Rightarrow A\right| \square \Gamma^{\prime}, \Gamma^{\prime \prime}, \Pi \Rightarrow$ $\square \Delta^{\prime}, \Delta^{\prime \prime}, \Sigma$, where $j \geqslant i$, \#top $(\rho)=\# \operatorname{top}(\pi)$ and \#top ${ }^{1}(\rho) \geqslant \# \operatorname{top}(\pi)$. By an application of $\square$ we get $\left.\right|^{j} \mathcal{G} \mid \square \Gamma^{\prime}, \Gamma^{\prime \prime}, \Pi \Rightarrow \square \Delta^{\prime}, \Delta^{\prime \prime}, \Sigma, \square A$.

THEOREM 3.5. Let $A$ be a modal formula with $\llbracket A \rrbracket=q$, then for every formula $B$ in $\mathcal{F}^{c}$, $\llbracket A \vee B \rrbracket \geqslant q$.

Proof. Let $\llbracket A \rrbracket=q$ and let us consider a derivation $\pi$ of $\stackrel{i}{\Rightarrow} A \vee B$. We can apply the rules of $\overline{\overline{\mathrm{HK}}}$ regardless of their order, so we decompose $\stackrel{i}{\Rightarrow} \Rightarrow A \vee B$ and we obtain derivations $\pi_{1}, \ldots, \pi_{n}$ of sequents $\stackrel{i_{1}}{\perp} \Gamma_{1} \Rightarrow A, \Delta_{1}, \ldots, \stackrel{i_{n}}{ } \Gamma_{n} \Rightarrow A, \Delta_{n}$ where each $\Gamma_{i}, \Delta_{i}$ is a multiset of atomic formulas obtained by the analysis of $B$ and $i=i_{1} \cdot \ldots \cdot i_{n}$.

We have

$$
\begin{aligned}
\operatorname{top}^{1}(A \vee B) & =\operatorname{top}^{1}\left(\pi_{1}\right) \uplus \cdots \uplus \operatorname{top}^{1}\left(\pi_{n}\right), \\
\operatorname{top}(A \vee B) & =\operatorname{top}\left(\pi_{1}\right) \uplus \cdots \uplus \operatorname{top}\left(\pi_{n}\right) .
\end{aligned}
$$

By Lemma 3.4, it easily follows that $\llbracket A \rrbracket \leqslant \llbracket A \vee B \rrbracket$.

Definition 6. Let $q \in \mathbb{Q}^{*}$. Then, the bounded consequence relation $\Gamma \vdash_{q} A$ holds just in case $\llbracket \wedge \Gamma \rightarrow A \rrbracket \geqslant q$. Accordingly, for every $q \in \mathbb{Q}^{*}$ we indicate with $\overline{\overline{\mathrm{HK}}}_{q}$ the logic whose set of theorems is $\{A \in \mathcal{F} \mid \llbracket A \rrbracket \geqslant q\}$.

THEOREM 3.6. For every $q \in \mathbb{Q}^{*}$ the bounded consequence relation $\vdash_{q}$ is reflexive and monotonic with respect to classical formulas.

Proof. Reflexivity follows from the fact that $\llbracket A \rightarrow A \rrbracket=1 \geqslant 1$. The monotonicity property follows from Lemma 3.5. 


$$
\begin{aligned}
D_{2}= & \left(\square\left(p_{1} \rightarrow p_{1}\right) \rightarrow \square\left(p_{2} \rightarrow p_{1}\right)\right) \vee \\
& \vee\left(\square\left(p_{1} \rightarrow p_{1}\right) \rightarrow \square\left(p_{3} \rightarrow p_{1}\right)\right) \vee \\
D_{1}=\left(\square\left(p_{1} \rightarrow p_{1}\right) \rightarrow \square\left(p_{2} \rightarrow p_{1}\right)\right) \vee & \vee\left(\square\left(p_{2} \rightarrow p_{2}\right) \rightarrow \square\left(p_{1} \rightarrow p_{2}\right)\right) \vee \\
\vee\left(\square\left(p_{2} \rightarrow p_{2}\right) \rightarrow \square\left(p_{1} \rightarrow p_{2}\right)\right) & \vee\left(\square\left(p_{2} \rightarrow p_{2}\right) \rightarrow \square\left(p_{3} \rightarrow p_{2}\right)\right) \vee \\
& \vee\left(\square\left(p_{3} \rightarrow p_{3}\right) \rightarrow \square\left(p_{1} \rightarrow p_{3}\right)\right) \vee \\
& \vee\left(\square\left(p_{3} \rightarrow p_{3}\right) \rightarrow \square\left(p_{2} \rightarrow p_{3}\right)\right)
\end{aligned}
$$

Fig. 4. Dugundji's formulas $D_{1}$ and $D_{2}$.

\section{§4. A new look at Dugundii's theorem.}

4.1. Dugundji's theorem. Intuitively, Dugundji's theorem put an end to the attempts to characterize modal logic by a truth-functional and finite-valued semantics, by stating the non-reducibility of the notion of possibility to an intermediate value between 0 and 1. Although the scope of the original formulation of Dugundji's theorem covers logics from $S 1$ to $S 5$, this result can also be extended to $K$ modulo slight modifications, as recently shown by Coniglio and Peron [8]. Let us first introduce the notions of matrix semantics and that of Dugundji's formula.

Definition 7 (Matrix, valuation, model). A matrix $\mathcal{M}$ on the language $\mathcal{F}$ consists of a triple $\langle M, \mathcal{O}, D\rangle$, where $M$ is a non-empty set of truth-values, $\mathcal{O}$ is a set of operations on $M$ interpreting the connectives of the language, and $D \subseteq M$ is a non-empty set of designated truth-values. A matrix $\mathcal{M}$ is said to be finite just in case the set $M$ is finite, otherwise it is infinite.

A valuation on a matrix $\mathcal{M}$ is a homomorphism (w.r.t. the operations in $\mathcal{O}$ ) $v: \mathcal{F} \rightarrow$ $M$. A formula $A$ is true, relative to $v$, if and only if $v(A) \in D$. If $A$ is true under any valuation $v$, then we say that $\mathcal{M}$ verifies $A$; in symbols, $\mathcal{M} \vDash A$. Accordingly, a matrix $\mathcal{M}$ is a model for the logic $K$ if, for any formula $A$, whenever HK proves $\Rightarrow A, A$ is verified in $\mathcal{M}$. A matrix $\mathcal{M}$ characterizes the logic $K$ if the following biconditional holds: HK proves the sequent $\Rightarrow A$ if, and only if, $\mathcal{M} \vDash A$.

Definition 8 (Dugundji's formulas ${ }^{3}$ ). For all $n \in \mathbb{N}$, the formula $D_{n}$ is defined as follows:

$$
D_{n}::=\bigvee\left(\square\left(p_{i} \rightarrow p_{i}\right) \rightarrow \square\left(p_{j} \rightarrow p_{i}\right)\right),
$$

where $1 \leqslant i, j \leqslant n+1$, and $i \neq j$.

ExAmple 4.1. Formulas $D_{1}$ and $D_{2}$ are shown in Figure 4.

THEOREM 4.1 (Dugundii's theorem for $K$ ). The modal logic K cannot be characterized by a finite matrix.

Proof. We propose a sketch of the proof as it has been provided in [8]. The whole argument takes shape by combining the two following results.

3 What we call here "Dugundji's formulas" is actually the hierarchy of formulas introduced by Coniglio and Peron in order to generalize further Dugundji's theorem. In his 1940 paper Dugundji identified a different sequence of formulas, inspired by the ones introduced by Gödel in 1932 to establish an analogous result for intuitionistic propositional logic (the birth of intermediate logics being a byproduct) [12]. 
(i) For any $n$-valued matrix $\mathcal{M}$, if $\mathcal{M}$ characterizes $K$, then $\mathcal{M}$ verifies the formula $D_{n}$. As a matter of fact, since there are exactly $n+1$ propositional variables occurring in $D_{n}$, for every valuation $v$ there will be $i, j$ such that $v\left(p_{i}\right)=v\left(p_{j}\right)$. Thence, upon considering that $\square\left(p_{i} \rightarrow p_{i}\right) \rightarrow \square\left(p_{i} \rightarrow p_{i}\right)$ is a theorem of $\mathrm{HK}$, it is immediate that $v\left(D_{n}\right) \in D$.

(ii) There exists a matrix with infinite elements $\mathcal{M}_{\infty}$ which is a model for $K$ and no $D_{n}$ is verified by $\mathcal{M}_{\infty}$. By definition, if $\Rightarrow A$ is provable in $\mathrm{HK}$, then $v(A) \in D$, for all $v$ on $\mathcal{M}_{\infty}$. That is, by contraposition, one has that the formula $D_{n}$ is not provable in $\mathrm{HK}$, for every $n \in \mathbb{N}$. Hence, $K$ cannot be characterized by a matrix with a finite number of elements.

4.2. Dugundji's theorem as a corollary of underivability. We turn now to a different proof of Dugundji's theorem which gives expression to an underivability result relative to $K$. In particular, the proof is obtained through a purely syntactic argument which exploits the analyticity of $\overline{\overline{\mathrm{HK}}}$, namely the fact that for each of the $\overline{\overline{\mathrm{HK}}}$ logical rules, the formulas in the premise(s) are always the subformula of some subformulas in the conclusion. This result allows us to streamline item $(i i)$ in the proof of Theorem 4.1 by avoiding any reference to the infinite matrix model $\mathcal{M}_{\infty}$. In short, for every $n \in \mathbb{N}$, $D_{n}$ is not derivable in HK. ${ }^{4}$

To reveal this, the following lemma is required.

Lemma 4.2. For any formula $A$, if $\mathrm{HK}$ proves $\mathcal{G} \mid A \rightarrow A, \Gamma \Rightarrow \Delta$, then $\mathrm{HK}$ also proves $\mathcal{G} \mid \Gamma \Rightarrow \Delta$.

Proof. Immediate by admissibility of the cut-rule in $\overline{\overline{\mathrm{HK}}}$ and so in $\mathrm{HK}$.

THEOREM 4.3. For every $n \in \mathbb{N}$, HK does not prove $\Rightarrow D_{n}$ or, equivalently, $\overline{\overline{\mathrm{HK}}}$ proves $\dashv \Rightarrow D_{n}$.

Proof. Let us assume, by contradiction, the derivability of the sequent $\Rightarrow D_{n}$ in $\mathrm{HK}$. By invertibility of the logical rules, the hypersequent:

$$
\begin{aligned}
\left(p_{1} \rightarrow p_{1}\right)^{n}, \ldots,\left(p_{n+1} \rightarrow p_{n+1}\right)^{n} & \Rightarrow p_{2} \rightarrow p_{1}|\cdots|\left(p_{1} \rightarrow p_{1}\right)^{n}, \ldots,\left(p_{n+1} \rightarrow p_{n+1}\right)^{n} \\
& \Rightarrow p_{n+1} \rightarrow p_{n}
\end{aligned}
$$

would be also derivable. By Lemma 4.2, one can finally get the derivability of the hypersequent:

$$
\Rightarrow p_{2} \rightarrow p_{1}|\cdots| \Rightarrow p_{n+1} \rightarrow p_{n} .
$$

By repeatedly analyzing this hypersequent by means of the $(\Rightarrow \rightarrow)$-rule one would obtain the provability of an instance of the $\overline{a x}$-rule.

REMARK 4. Theorem 4.3 relies on the fact that cut is admissible in HK, which is the deductive upper bound of the chain of extended systems $\overline{\overline{\mathrm{HK}}}_{q}$. This implies that the addition of arbitrary identities in the left-hand side of the sequent sign does not affect the derivability relation. However, this is not true of bounded systems, since bounded consequence relations are not transitive.

4 Of course, the underivability of Dugundji's formulas can indeed be shown also by means of a more standard sequent calculus or of a suitable tableaux system for $K$. However, such systems do not allow for a fractional reading of the theorem. 
Theorem 4.3 provides the ground for an alternative proof of Dugundji's theorem, whereby an infinite matrix is not required.

THEOREM 4.4 (Dugundji's theorem for $K$ : fractional version). The modal logic $K$ cannot be characterized by a finite matrix.

Proof. Suppose there is an $n$-valued matrix $\mathcal{M}$ which characterizes $K$, i.e., $\mathcal{M} \vDash A$ iff the sequent $\Rightarrow A$ is provable in HK. As in the proof of Theorem 4.1, $\mathcal{M}$ satisfies Dugundji's formula $D_{n}$. Therefore, by hypothesis $K \vdash D_{n}$, but $\mathrm{HK} \nvdash \Rightarrow D_{n}$ against Theorem 4.3.

4.3. A function for Dugundji's formula. Finally, we define the function $\varphi: \mathbb{N}^{+} \mapsto \mathbb{Q}^{*}$ by putting $\varphi(n)=\llbracket D_{n} \rrbracket$, i.e., $\varphi$ yields a complete description of the fractional values assigned with every formula $D_{n}$. A simple calculation reveals that the number of disjuncts in any formula $D_{n}$ is exactly $n(n+1)$. We can now prove the following:

THEOREM 4.5. $\varphi(n)=1-\frac{1}{2^{2 n^{2}(n+1)}}$, for any $n \in \mathbb{N}^{+}$.

Proof. The proof amounts to showing that $\llbracket D_{n} \rrbracket=1-\frac{1}{2^{2 n^{2}(n+1)}}$, for any Dugundji's formula $D_{n}$. In particular, for every $n \in \mathbb{N}$, we apply rules $\Rightarrow \vee, \Rightarrow \rightarrow$ and $\square$ to decompose the formula $D_{n}$ into a hypersequent formed by a multiset of sequents each one of them having the following general form:

$$
\left(p_{1} \rightarrow p_{1}\right)^{n}, \ldots,\left(p_{n+1} \rightarrow p_{n+1}\right)^{n}, p_{j} \Rightarrow p_{i},
$$

where $i \neq j$ and $1 \leqslant i, j \leqslant n+1$. The number of components is $n^{2}+n$ (we omit the component containing only boxed formulas in the antecedent, which can be removed without affecting the counting of the top-hypersequents). Since the number of implications in the antecedent of every component is $(n+1) \cdot n$, the total number of implications to be analyzed in the hypersequent will be $(n+1) \cdot n \cdot\left(n^{2}+n\right)$. Therefore, the number of top-hypersequents is $2^{(n+1)^{2} \cdot n^{2}}$.

We now need to compute the number of non-axiomatic top-sequents. Given a component:

$$
\left(p_{1} \rightarrow p_{1}\right)^{n}, \ldots,\left(p_{n+1} \rightarrow p_{n+1}\right)^{n}, p_{j} \Rightarrow p_{i},
$$

there are $n(n-1)$ implications whose analysis does not lead to any axiomatic tophypersequent, namely the implications $p_{k} \rightarrow p_{k}$, where $k \neq j, i$. Therefore, we can conclude that the hypersequent whose analysis will lead to complementary axioms has every component of the form:

$$
\left(p_{k_{1}} \rightarrow p_{k_{1}}\right)^{n}, \ldots,\left(p_{k_{n-1}} \rightarrow p_{k_{n-1}}\right)^{n}, p_{j}^{n+1} \Rightarrow p_{i}^{n+1},
$$

where $\left\{k_{1}, \ldots, k_{n-1}\right\}=\{1, \ldots, n+1\}-\{j, i\}$. Since the components are $n^{2}+n$, the number of complementary top-hypersequents is $2^{(n-1) \cdot n \cdot\left(n^{2}+n\right)}=2^{n^{4}-n^{2}}$.

As a consequence, we can state that for every $n \geqslant 1$ :

$$
\llbracket D_{n} \rrbracket=\frac{\# \operatorname{top}\left(D_{n}\right)-\# \operatorname{top}^{0}\left(D_{n}\right)}{\# \operatorname{top}\left(D_{n}\right)}=\frac{2^{(n+1)^{2} \cdot n^{2}}-2^{n^{4}-n^{2}}}{2^{(n+1)^{2} \cdot n^{2}}}=1-\frac{1}{2^{2 n^{2}(n+1)}} .
$$


ExAmple 4.2. $\llbracket D_{1} \rrbracket=1-\frac{1}{2^{4}}=0.9375$ and $\llbracket D_{2} \rrbracket=1-\frac{1}{2^{24}}=0.999999940395355$.

The next results offer yet another perspective on Dugundji's theorem through fractional semantics.

Proposition 4.6. For any $m, n \in \mathbb{N}^{+}$such that $n<m$, we have $0<\llbracket D_{n} \rrbracket<\llbracket D_{m} \rrbracket$.

Proof. It suffices to observe that if $n<m$, then $\left(\frac{1}{4}\right)^{m^{3}+m^{2}}<\left(\frac{1}{4}\right)^{n^{3}+n^{2}}$ and thus $1-$ $\left(\frac{1}{4}\right)^{n^{3}+n^{2}}<1-\left(\frac{1}{4}\right)^{m^{3}+m^{2}}$.

Corollary 4.7. For every $n \in \mathbb{N}$, HK does not prove $\Rightarrow D_{n}$.

Proof. Straightforwardly by Proposition 4.6.

Proposition 4.6 marks an informational refinement with respect to standard Kripkean semantics. In fact, the fractional approach allows for a fine-grained analysis of $K$-invalidities. In particular, the sequence of rationals $\llbracket D_{0} \rrbracket, \llbracket D_{1} \rrbracket, \ldots$ generated by the fractional evaluation of Dugundji's formulas proves rapidly approaching near to its limit 1 without ever reaching it.

§5. Concluding remarks. In this paper, we syntactically framed the basic modal logic $K$ within the bilateral hypersequent calculus $\overline{\overline{\mathrm{HK}}}$ satisfying stability and invertibility of the logical rules. We showed how to use $\overline{\overline{\mathrm{HK}}}$ in order to fractionally interpret modal formulas: any modal formula $A$ is evaluated in terms of the ratio between axiomatic top-hypersequents and the totality of the top-hypersequents figuring in any derivation of $A$. Such a result has been achieved by generalizing and, then, adapting a methodology originally designed for classical propositional logic. Moreover, we argued that fractional semantics also conveys a different way of thinking about proof-theoretic semantics due to its commitment to the primacy of the notion of (analytic) proof. Here, in particular, our semantic treatment of $K$ should be understood as a step toward a proof-theoretic semantics in modal settings (for other attempts in this direction see $[19,23]$ ).

In conclusion, we would like to hint at some themes for further research. First of all, it could be interesting to extend the fractional approach so as to include other systems of modal logic. Such an extension can be conducted along two different directions. On the one hand, one could take into account non-normal modal logics weaker than $K$, not characterized by Kripkean semantics, as well as stronger logics, such as $S 4$, serving as base of epistemic logics. On the other hand, it would be tempting to explore the relation with other proof-theoretic frameworks. We are inclined to think, in fact, that the fractional interpretation is invariant under other calculi (such as nested sequents, labeled sequents, and prefixed tableaux), as long as these fulfill the three requirements of bilateralism, invertibility and stability. Moreover, the properties of the system $\overline{\overline{\mathrm{HK}}}$ (and its possible extensions) could be also investigated from a more traditional prooftheoretic perspective by presenting a Gentzen-style cut-elimination algorithm.

Lastly, it is worth observing that fractional semantics can be also conceived as an attempt to introduce a measure of invalidity, blurring the rigid partition between valid and invalid formulas. In this respect, our treatment could be fruitfully related to the 
work in [15] in which some semantic criteria are introduced in order to measure the inconsistency of modal formulas.

Acknowledgment. We wish to thank two anonymous referees for their useful comments which helped us to improve the overall quality of the paper.

\section{BIBLIOGRAPHY}

[1] Avron, A. (1987). A constructive analysis of RM. The Journal of Symbolic Logic, 52(4), 939-951.

[2] (1991). Hypersequents, logical consequence and intermediate logics for concurrency. Annals of Mathematics and Artificial Intelligence, 4(3-4), 225-248.

[3] — (1996). The method of hypersequents in the proof theory of propositional non-classical logics. In Hodges, W., Hyland, M., Steinhorn, C., and Truss, J., editors. Logic: From Foundations to Applications. Oxford: Clarendon Press, pp. 1-32.

[4] Bendall, K. (1979). Negation as a sign of negative judgment. Notre Dame Journal of Formal Logic, 20(1), 68-76.

[5] Blackburn, P., de Rijke, M., \& Venema, Y. (2001). Modal Logic. Cambridge Tracts in Theoretical Computer Science, Vol. 53. Cambridge: Cambridge University Press.

[6] Brünnler, K. (2009). Deep sequent systems for modal logic. Archive for Mathematical Logic, 48(6), 551-577.

[7] Chagrov, A., \& Zakharyaschev, M. (1997). Modal Logic. Oxford Logic Guides, Vol. 35. New York: Oxford University Press.

[8] Coniglio, M. E., \& Peron, N. M. (2014). Dugundji's theorem revisited. Logica Universalis, 8(3-4), 407-422.

[9] Dugundji, J. (1940). Note on a property of matrices for Lewis and Langford's calculi of propositions. The Journal of Symbolic Logic, 5(4), 150-151.

[10] Francez, N. (2014). Bilateralism in proof-theoretic semantics. Journal of Philosophical Logic, 43(2-3), 239-259.

[11] - (2015). Proof-Theoretic Semantics. London: College Publications.

[12] Gödel, K. (1932). Zum intuitionistischen Aussagenkalkül. Anzeiger der Akademie der Wissenschaften Wien, mathematisch-naturwissenschaftiche Klasse, 69 , 65-66.

[13] Goranko, V. (2019). Hybrid deduction-refutation systems. Axioms, 8(4), 118.

[14] Goranko, V., Pulcini, G., \& Skura, T. (2020). Refutation systems: An overview and some applications to philosophical logics. In Liu, F., Ono, H., and Yu, J., editors. Knowledge, Proof and Dynamics. Singapore: Springer, pp. 173-197.

[15] Grant, J. (2020). Measuring inconsistency in some logics with modal operators. Studia Logica, 109, 581-605.

[16] Ketonen, O. (1944). Untersuchungen zum Prädikatenkalkül. Annales Academiae Scientiarum Fennicae. Series A, I, 1-77.

[17] Kleene, S. C. (1967). Mathematical Logic. London: John Wiley \& Sons.

[18] Kürbis, N. (2015). Proof-theoretic semantics, a problem with negation and prospects for modality. Journal of Philosophical Logic, 44(6), 713-727.

[19] Leszcczyńska, D. (2004). Socratic proofs for some normal modal propositional logics. Logique et Analyse, 47, 259-285. 
[20] Mints, G. (1992a). Lewis' systems and system T (1965-1973). In Selected Papers in Proof Theory. Pittsburgh: Bibliopolis, pp. 221-294.

[21] - (1992b). A Short Introduction to Modal Logic. CLSI Lecture Notes, Vol. 30. Stanford: Center for the Study of Language (CSLI).

[22] Negri, S. (2005). Proof analysis in modal logic. Journal of Philosophical Logic, 34(507), 507-544.

[23] Parisi, A. (2020). A hypersequent solution to the inferentialist problem of modality. Erkenntnis, https://doi.org/10.1007/s10670-020-00264-x.

[24] Piazza, M., \& Pulcini, G. (2020). Fractional semantics for classical logic. The Review of Symbolic Logic, 13(4), 810-828.

[25] Piecha, T., \& Schroeder-Heister, P. (2016). Advances in Proof-Theoretic Semantics. Cham: Springer.

[26] Poggiolesi, F. (2010). Display calculi and other modal calculi: A comparison. Synthese, 173(3), 259-279.

[27] Pottinger, G. (1983). Uniform, cut-free formulations of T, S4 and S5. Journal of Symbolic Logic, $\mathbf{4 8}(3), 900$.

[28] Pulcini, G., \& Varzi, A. Classical logic through rejection and refutation. In Fitting, M., editor. Landscapes in Logic, Vol. 2. College Publications, to appear.

[29] Rumfitt, I. (2000). 'Yes and no'. Mind, 109(436), 781-823.

[30] Troelstra, A. S., \& Schwichtenberg, H. (2000). Basic Proof Theory. Cambridge Tracts in Theoretical Computer Science, Vol. 43. Cambridge: Cambridge University Press.

[31] Viganò, L. (2000). Labelled Non-classical Logics. Dordrecht: Kluwer Academic.

[32] Wansing, H. (2000). The idea of a proof-theoretic semantics and the meaning of the logical operations. Studia Logica, 64(1), 3-20.

[33] _ (2002). Sequent systems for modal logics. In Gabbay, D. M., and Guenthner, F., editors. Handbook of Philosophical Logic, Vol. 8. Dordrecht: Springer, pp. 61-145.

[34] —. (2013). Displaying Modal Logic, Vol. 3. Dordrecht: Springer Science \& Business Media.

[35] - (2017). A more general general proof theory. Journal of Applied Logic, 25, $23-46$.

\author{
CLASSE DI LETTERE E FILOSOFIA \\ SCUOLA NORMALE SUPERIORE DI PISA \\ PISA, ITALY \\ E-mail: mario.piazza@sns.it \\ E-mail:matteo.tesi@sns.it \\ DIPARTIMENTO DI STUDI LETTERARI, FILOSOFICI E DI STORIA DELL'ARTE \\ UNIVERSITÀ DI ROMA "TOR VERGATA" \\ ROME, ITALY \\ E-mail: gabriele.pulcini@uniroma2.it
}

